\title{
3D Global Weak-Form Mesh-Free Method for Acoustic Attenuation Analysis of Expansion Chambers
}

\author{
Chun-Hui Fang, ${ }^{1}$ Cheng-Yang Liu, ${ }^{2}$ and Zhi Fang $\mathbb{D}^{3}$ \\ ${ }^{1}$ Yantai Automobile Engineering Professional College, Yantai, Shandong 265500 430074, China \\ ${ }^{2}$ China Ship Development and Design Center, Wuhan, Hubei 430064, China \\ ${ }^{3}$ School of Naval Architecture and Ocean Engineering, Huazhong University of Science and Technology, Wuhan, \\ Hubei 430074, China \\ Correspondence should be addressed to Zhi Fang; fangzhi@hust.edu.cn
}

Received 5 June 2020; Revised 10 July 2020; Accepted 26 July 2020; Published 9 September 2020

Academic Editor: Chao Tao

Copyright (c) 2020 Chun-Hui Fang et al. This is an open access article distributed under the Creative Commons Attribution License, which permits unrestricted use, distribution, and reproduction in any medium, provided the original work is properly cited.

\begin{abstract}
In order to avoid the dependence of mesh method on grids, a 3D global weak-form mesh-free method (MFM) is applied to study the three-dimensional acoustic characteristics of silencers. For the expansion chamber silencers, the 3D acoustic modes are extracted and the transmission loss results are computed by using the 3D global weak-form (MFM), which is based on the radial basis function point interpolation method (RPIM) for calculating the shape functions and Galerkin method for discretizing the system equation. The first 15 order 3D acoustic modes and TL results of a special expansion chamber silencer are presented to validate the computational accuracy of the proposed technique, and the relative errors are controlled within $0.5 \%$ by comparing with the 3D finite element method (FEF) calculations. Additionally, the effects of axial modes on the acoustic characteristics are investigated, and the pass through frequencies can be eliminated to enhance the acoustic attenuation performance by locating the side branch outlet on the nodal lines of axial modes.
\end{abstract}

\section{Introduction}

Silencers are widely used in engineering applications in cars, aircrafts, ships, and submarines. The acoustic modes have significant effects on acoustic characteristics of silencers. The configuration of silencers can be improved to optimize the acoustic attenuation performance according to the acoustic modal characteristics. In recent years, many methods including analytical and numerical methods have been developed to predict the acoustic modes and transmission loss of silencers. The two-dimensional transversal modes of regular cross sections of silencers are calculated by using the analytical method in [1-4], and the transmission loss results are calculated by using the mode-matching method. The analytical methods have the advantage of high computational accuracy and efficiency but are confined to relatively simple and regular configurations. In [5-12], the two-dimensional transversal modes of the expansion chamber silencers and the perforated tube silencers with arbitrary cross section are calculated by using the $2 \mathrm{D}$ finite element method, and the effects of the structure parameters of chambers and perforated tubes on the transversal modes are investigated $[9,10]$. The finite element method can be used for the acoustic modes of any complex silencers, but the numerical accuracy is largely determined by the quantity and quality of the mesh elements and are highly timeconsuming when the size of the silencer is large or the computational frequency range is wide. For this reason, the mesh-free methods are proposed to calculate the 2D acoustic problems [13-20].

Mesh-free methods (MFMs) have several advantages over the FEM, and over the past decades, many MFMs have been developed to study acoustic eigen problems. The method of fundamental solution (MFS) [13] is investigated to calculate the eigenvalues of the Helmholtz equation in a plane with homogeneous Dirichlet boundary conditions, and it can be concluded that the MFS exhibited much faster convergence than did the boundary integral equation method. 
The radial point interpolation method (RPIM) for the Helmholtz equation in the $2 \mathrm{D}$ case is studied by Wentreodt [14]. Three different radial basis function point interpolation methods (RPIMs) were studied in that work, and all of them showed a significant reduction of the dispersion error compared with that of the FEM. The dual reciprocity hybrid boundary node method (DRHBNM), combining the hybrid boundary node method (HBNM) with the dual reciprocity method (DRM), is applied for solving acoustic eigenvalue problems by Li et al. [15]. The numerical examples of several acoustic cavities with different boundary conditions were provided, and suitable accuracy was demonstrated in that paper. The meshless Galerkin least-squares (MGLS) method [16] is proposed to a $2 \mathrm{D}$ acoustic problems, and the numerical examples of an L-shaped cavity demonstrated the MGLS method had higher computational efficiency than the EFGM. The cell-based smoothed radial point interpolation method (CS-RPIM) for 2D acoustic problems was addresses in [17], and the numerical results proved that the CS-RPIM achieved more accurate results and higher convergence rates than did the FEM. A method based on a radial basis function (RBF) and collocation method for the wave propagation problem was introduced by Wang et al. [18], and an eigenvalue analysis was implemented to evaluate the effectiveness and accuracy of the proposed method. Fang et al. $[19,20]$ proposed a combined MFM and mode-matching approach to predict the transmission loss of silencers. In [19], the Hermite radial point interpolation collocation (Hermite-RPI-collocation) method was applied to solve the transversal eigenequations of expansion chamber silencers. The authors noted that the HRPIC method was more efficient than the FEM; however, the calculation precision was sensitive to the shape parameters of RBF, especially for problems with Neumann boundary conditions. Then, in [20], the weak-form MFM based on an integration scheme was proposed to derive the transversal modes of perforated tube silencers. The radial point interpolation method (RPIM) was used to create the shape functions, and the Galerkin method was employed to obtain the weak formulation of the transversal governing equations. It can be concluded that the MFMs for solving the transversal eigenmodes are independent of the element topology and require fewer nodes and consume less time than does the FEM.

All the above calculations are two-dimensional, only the transversal modes are calculated, and the $3 \mathrm{D}$ modal effects are not analyzed. In this paper, the three-dimensional global weak-form mesh-free method is used to calculate the threedimensional acoustic modes and transmission loss of simple expansion chamber silencers. In Section 3, the radial basis function point interpolation method is used to solve the three-dimensional mesh-free function, the Galerkin weighted residual method is used to discrete the system equation, and the three-dimensional global weak mesh-free method is used to solve the 3D acoustic modes and TL. Then, the acoustic modes and TL predictions for expansion chamber silencer are compared to verify the calculation precision of the proposed technique in Section 4. Finally, the influence of axial mode on the acoustic attenuation performance is investigated in Section 5.

\section{Three-Dimensional Sound Propagation in an Expansion Chamber}

Considering an expansion chamber silencer with an arbitrarily shaped cross section as shown in Figure 1, the propagation of sound pressure inside the ducts is governed by the acoustic wave equation, which is derived from the ideal gas law and the laws of conservation of mass and momentum. For a time harmonic wave, the three-dimensional acoustic wave equations for the expansion chamber silencer can be expressed as the following Helmholtz equations [21]:

$$
\nabla^{2} p+k^{2} p=0
$$

where $\nabla^{2}=\partial^{2} / \partial x^{2}+\partial^{2} / \partial y^{2}+\partial^{2} / \partial z^{2}$ is the Laplacian operator in the three-dimensional Cartesian coordinate system, and $p$ is the sound pressure. $k=\left(\omega / c_{0}\right)$ is the wavenumber in air, and $\omega$ is the angular frequency, and $c_{0}$ is the speed of sound in air.

Three boundary conditions are used to model the expansion charmer silences represented in Figure 1.

(1) Rigid wall boundary $\left(S_{r}\right)$ : the normal acoustic velocity vanishes at the side wall of the silencer:

$$
\left.\frac{\partial p}{\partial n}\right|_{S_{r}}=0
$$

(2) Imposed normal particle velocity boundary $\left(S_{v}\right)$ : to represent the plane wave excitation incident to the inlet duct of the expansion chamber, this boundary condition is employed at the upstream of the silencer; the equation for this boundary is

$$
\left.\frac{\partial p}{\partial n}\right|_{S_{v}}=-j \rho_{0} \omega u_{n}
$$

where $u_{n}$ is the prescribed particle velocity normal to and away from the surface.

(3) Imposed normal acoustic impedance boundary $\left(S_{z}\right)$ : on this boundary, the nonflection boundary is simulated by defining the acoustic impedance at the downstream of the silencers as the acoustic impedance of the air; this is achieved by the following:

$$
\left.\frac{\partial p}{\partial n}\right|_{S_{z}}=-j \rho_{0} \omega \frac{p}{z_{0}}
$$

where $z_{0}=\rho_{0} c_{0}$ is the acoustic impedance of the air.

\section{Three-Dimensional Weak-Form MFM for Expansion Chamber Silencer}

The 3D mesh-free method model of the expansion chamber silencer is obtained by discretizing the Helmholtz equations (1) over the numerical domain using the mesh-free shape function. After introducing the boundary conditions ((2)-(4)) into the weak form of the governing equation (1), 


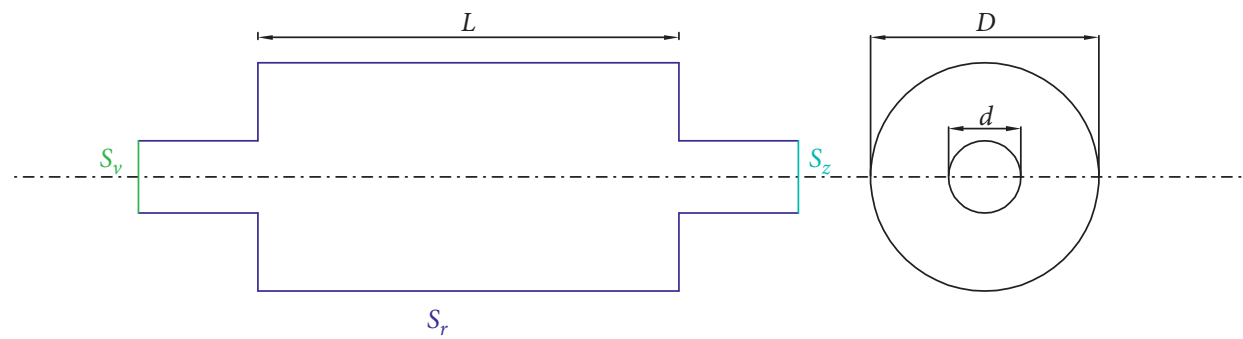

FIgURe 1: Description of the boundary conditions used for modelling the expansion chamber silencer: $S_{r}$ is the rigid wall boundary, $S_{v}$ is the imposed normal particle velocity boundary, and $S_{z}$ is the imposed normal acoustic impedance boundary.

the system of equations representing the MFM model can be derived.

\subsection{Construction of the 3D Mesh-Free Shape Function.} The RBF is applied to form the shape function based on the RPIM. The distribution of field points inside the support domain can be random for convenience [22]. For each computational point $\mathbf{x}^{T}=[x, y, z]$, an approximation $p^{h}(\mathbf{x})$ of the acoustic pressure can be written as a linear combination of RBFs:

$$
p^{h}(\mathbf{x})=\sum_{i=1}^{n} R_{i}(\mathbf{x}) a_{i}+\sum_{k=1}^{m} q_{k}(\mathbf{x}) c_{k}=\mathbf{B}^{T} \mathbf{a}_{0} .
$$

Here, $R_{i}(\mathbf{x})$ is the RBF, and $q_{k}(\mathbf{x})$ is an unknown polynomial. $n$ and $m$ pertain to the RBF and polynomials, and $a_{i}$ and $c_{k}$ are the corresponding coefficients. The matrices in (5) can be expressed as follows:

$$
\begin{aligned}
& R_{i}(\mathbf{x})=R_{i}(x, y, z)=\sqrt{r_{i}^{2}+\left(a_{c} d_{c}\right)^{2}}, \\
& \mathbf{B}^{T}=\left[\begin{array}{llllllll}
R_{1} & \cdots & R_{n} & 1 & x & y & \cdots & q_{m}(\mathbf{x})
\end{array}\right], \\
& \mathbf{a}_{0}=\left[\begin{array}{llllll}
a_{1} & \cdots & a_{n} & c_{1} & \cdots & c_{m}
\end{array}\right]^{T} .
\end{aligned}
$$

To derive the coefficients $a_{i}$ and $c_{k}$ in (5), a support domain of computational point $\mathbf{X}$ is defined, in which $n$ field nodes are contained. The size of the support domain is determined by $d_{s}=\alpha_{s} d_{c}$, where $\alpha_{s}$ is a nondimensional parameter. $d_{c}$ is the average distance of field nodes in the support domain. $R_{i}\left(k_{i}\right)$ and $\alpha_{c}$ in the RBF are dimensionless shape parameters. It was previously shown that choosing appropriate parameters is important for optimizing simulations [23]. The distance $r_{k}$ between the nodes $i$ and $k$ is defined as

$$
r_{k}=\sqrt{\left(x_{i}-x_{k}\right)^{2}+\left(y_{i}-y_{k}\right)^{2}+\left(z_{i}-z_{k}\right)^{2}} .
$$

Each node contained in the support domain must satisfy (5). In addition, to obtain the unique solution, the following constraint equations are applied to the polynomial terms:

$$
\sum_{i=1}^{n} q_{k}\left(\mathbf{x}_{i}\right) a_{i}=0, \quad k=1,2, \ldots, m .
$$

Then, the following matrix equations can be derived:

$$
\begin{aligned}
& \widetilde{\mathbf{p}}=\left[\frac{\mathbf{p}}{\mathbf{0}}\right]=\left[\begin{array}{ll}
\mathbf{R}_{0} & \mathbf{Q}_{m} \\
\mathbf{Q}_{m}^{T} & 0
\end{array}\right]\left[\begin{array}{l}
\mathbf{a} \\
\mathbf{c}
\end{array}\right]=\mathbf{G a}_{0}, \\
& \mathbf{R}_{0}=\left[\begin{array}{cccc}
R_{1}\left(\mathbf{x}_{1}\right) & R_{2}\left(\mathbf{x}_{2}\right) & \cdots & R_{n}\left(\mathbf{x}_{1}\right) \\
R_{1}\left(\mathbf{x}_{2}\right) & R_{2}\left(\mathbf{x}_{2}\right) & \cdots & R_{n}\left(\mathbf{x}_{2}\right) \\
\vdots & \vdots & \ddots & \vdots \\
R_{1}\left(\mathbf{x}_{n}\right) & R_{2}\left(\mathbf{x}_{n}\right) & \cdots & R_{n}\left(\mathbf{x}_{n}\right)
\end{array}\right]_{(n \times n)}, \\
& \mathbf{Q}_{m}=\left[\begin{array}{cccccc}
1 & x_{1} & y_{1} & z_{1} & \cdots & q_{m}\left(\mathbf{x}_{1}\right) \\
1 & x_{2} & y_{2} & z_{3} & \cdots & q_{m}\left(\mathbf{x}_{2}\right) \\
\vdots & \vdots & & & \ddots & \vdots \\
1 & x_{n} & y_{n} & z_{n} & \cdots & q_{m}\left(\mathbf{x}_{n}\right)
\end{array}\right]_{(n \times m)} \\
& \widetilde{\mathbf{p}}=\left[\mathbf{p}\left(\mathbf{x}_{1}\right), \ldots, \mathbf{p}\left(\mathbf{x}_{n}\right), 0, \ldots, 0\right]^{T} .
\end{aligned}
$$

Clearly, matrix $\mathbf{G}$ is symmetric and invertible. Thus, the coefficient matrix $\mathbf{a}_{0}$ can be obtained by solving (11):

$$
\mathbf{a}_{0}=\mathbf{G}^{-1} \tilde{\mathbf{p}}
$$

By substituting (15) into (5), we obtain

$$
\mathbf{p}^{h}(\mathbf{x})=\mathbf{B}^{T} \mathbf{a}_{0}=\mathbf{B}^{T} \mathbf{G}^{-1} \widetilde{\mathbf{p}}=\widetilde{\Phi}^{T} \widetilde{\mathbf{p}},
$$

where $\widetilde{\Phi}$ is a shape function that can be described as

$$
\boldsymbol{\varphi}^{\mathrm{T}}=\mathbf{B}^{T} \mathbf{G}^{-1}=\left[\Phi_{1}, \Phi_{2}, \ldots, \Phi_{n}, \Phi_{1}^{q}, \ldots, \Phi_{m}^{q}\right] .
$$

The RPIM shape functions $\Phi$ related to nodal sound pressures are expressed as

$$
\boldsymbol{\Phi}^{\mathrm{T}}=\left[\Phi_{1}, \Phi_{2}, \ldots, \Phi_{n}\right] .
$$

Finally, the transversal sound pressure $p^{h}(\mathbf{x})$ of the computational point is derived:

$$
p^{h}(\mathbf{x})=\sum_{i=1}^{n} \Phi_{i} p_{i}=\Phi^{\mathrm{T}} \mathbf{p}
$$

where $i$ denotes the $i^{\text {th }}$ node in the support domain, and $p_{i}$ is defined as the sound pressure for the $i^{\text {th }}$ node.

3.2. Galerkin Method for Weak Formulation. The method of weighted residuals is used to obtain the weak-form MFM 
formulation of the governing equation (1) and the boundary conditions (2)-(4). The residuals of the eigenequation and boundary conditions are multiplied by weighting functions and integrated over their respective surfaces. The Galerkin method is used in this paper, and the weighting functions are the same as the shape function $\Phi$. Finally, the system of equations representing the MFM model takes the following form:

$$
\left\{[\mathbf{K}]-k_{0}^{2}[\mathbf{M}]+\frac{j \rho_{0} \omega}{z}[\mathbf{Z}]\right\}\{\mathbf{p}\}=-j \rho_{0} \omega u_{n}\{\mathbf{F}\},
$$

where

$$
\begin{aligned}
& {[\mathbf{K}]=\int_{V}\{\nabla \Phi\}\{\nabla \Phi\}^{T} d V,} \\
& {[\mathbf{M}]=\int_{V}\{N\}\{N\}^{T} d V,} \\
& {[\mathbf{Z}]=\int_{S_{z}}\{\Phi\}\{\Phi\}^{T} d S,} \\
& {[\mathbf{F}]=\int_{S_{v}}\{\Phi\} d S,}
\end{aligned}
$$

where $\mathbf{K}$ is the stiffness, $\mathbf{M}$ is the mass, $\mathbf{Z}$ is the impedance, and $\mathbf{F}$ is the forcing matrices.

\subsection{Hammer Integration Scheme for Numerical Integration.}

To obtain the system matrices in (21), an integration grid is required to carry out the numerical integration over the domain and its boundary. Regularly shaped (e.g., tetrahedron or hexahedron) cells are typically chosen in the weakform MFM, since it is difficult to divide the expansion chambers into regular hexahedral meshes; therefore in this paper, a tetrahedron with five Hammer points is used.

For each Hammer point, a support domain is defined, and the shape functions are computed individually. The integration over a cell is implemented using

$$
\mathbf{K}=\sum_{e} \int_{V_{e}}\{\nabla \Phi\}\{\nabla \Phi\}^{T} d V_{e}=\sum_{e} \sum_{Q=1}^{n_{Q}} \omega_{Q} \operatorname{det}\left(\mathbf{J}_{e}\right)(\nabla \Phi)_{e}(\nabla \Phi)_{e}^{T},
$$

$\mathrm{M}=\sum_{e} \int_{V_{e}}\{\Phi\}\{\Phi\}^{T} d V_{e}=\sum_{e} \sum_{Q=1}^{n_{Q}} \omega_{Q} \operatorname{det}\left(\mathbf{J}_{e}\right)(\Phi)_{e}(\Phi)_{e}^{T}$,

$$
\mathbf{Z}=\sum_{e} \int_{S_{e}}\{\Phi\}\{\Phi\}^{T} d S_{e}=\sum_{e} \sum_{Q=1}^{n_{Q}} \omega_{Q} \operatorname{det}\left(\mathbf{J}_{e}\right)(\Phi)_{e}(\Phi)_{e}^{T},
$$

$$
\mathbf{F}=\sum_{e} \int_{S_{e}}\{\Phi\} d S_{e}=\sum_{e} \sum_{Q=1}^{n_{Q}} \omega_{Q} \operatorname{det}\left(\mathbf{J}_{e}\right)(\Phi)_{e},
$$

where $n_{Q}$ is the number of Hammer points in cell $e, w_{Q}$ denotes the associated Hammer weight, and $\mathbf{J}_{e}$ is the $\mathrm{Ja}$ cobian matrix of cell $e$.

The weight coefficient and integration point coordinates for the $3 \mathrm{D}$ Hammer integration is listed in Table 1.
3.4. Eigenmodes and Transmission Loss Calculation. The sound pressure of every node can be obtained by solving (20), and then the transmission loss of expansion chamber silencer can be derived by using the following form:

$$
\mathrm{TL}=20 \log _{10}\left\{{\frac{S_{1}}{S_{2}}}^{1 / 2}\left|\frac{p_{1}+\rho_{0} c_{0} v_{1}}{2 p_{2}}\right|\right\}
$$

where $v_{1}$ is the defined particle velocity at the inlet. $p_{1}$ and $p_{2}$ are the sound pressure at the inlet and outlet of the silencer, which can be obtained by using the weak-form MFM.

When solving the eigenmodes of the expansion chamber, the boundary condition is rigid wall boundary as (2), and the MFM equation of the system is simplified as

$$
\left\{[\mathbf{K}]-k_{0}^{2}[\mathbf{M}]\right\}\{\mathrm{p}\}=\{\mathbf{0}\} .
$$

Supposing that $n$ field nodes are contained in the transversal section, the axial wavenumber $\mathbf{k}_{z}$ and eigenvector matrix $\mathbf{X}_{x y}$ under every excitation frequency can be obtained by solving (27). The dimensions of $\mathbf{k}_{z}$ and $\mathbf{X}_{x y}$ are $n \times 1$ and $n \times n$, respectively.

\section{Validation of the MFM Model for Acoustic Mode and TL Calculation}

4.1. Expansion Chamber Silencer. In order to validate the computational accuracy, the first 15 order higher modal frequencies and modal shapes of the simple expansion chamber silencer shown in Figure 1 are calculated by using the $3 \mathrm{D}$ weak-form MFM and comparing with the simulations from finite element method. The physical properties of the silencer used in the validation are the diameter of the chamber $D=0.1532 \mathrm{~m}$, length of the chamber $L=0.2823 \mathrm{~m}$, and diameter of the inlet/outlet duct $d=0.0486 \mathrm{~m}$. Since the dimension of inlet/outlet is much smaller than that of the chamber, in the high frequency range (below $4150 \mathrm{~Hz}$ ) the sound filed is plane wave propagation, therefore, in the interested frequency range $(0-3200 \mathrm{~Hz})$, the higher-order modes of the silencer are only excited in the chamber. Consequently, the $3 \mathrm{D}$ acoustic modes of the chamber are calculated in this paper. The distributions of the background mesh and the field nodes for calculating acoustic modes are shown in Figure 2.

The field node distributions are obtained by using MATLAB codes, and the background meshes used in the weak-form MFM are created by the software package ANSYS, which are independent of the field nodes.

Table 2 gives the first 15 order higher modal frequencies from the 3D weak-from MFM and 3D FEM. From Table 2, it can be seen that the relative errors of the acoustic modes are less than $0.5 \%$, which validates the application of the $3 \mathrm{D}$ weak-form MFM for the acoustic mode calculation.

All the modal shapes are displayed in Figure 3. In the modal shapes, the line for expressing the zero pressures is called nodal line. 3D acoustic modes can be expressed by the number of circumferential nodal line $m$, radial nodal line $n$, and axial nodal line $z$, namely, $(m, n, z)$. 
TABLE 1: Weight coefficient and integration point coordinates for the Hammer integration.

\begin{tabular}{lccc}
\hline Number of integration points & Weight coefficient & Integration point coordinate & Order of precision \\
\hline 1 & A 1 & $(1 / 4),(1 / 4),(1 / 4),(1 / 4)$ & 1 \\
4 & B2 & $a=0.58541020$ & 2 \\
5 & A1 $=-(4 / 5)$ & $b=0.13819660$ & 3 \\
\hline
\end{tabular}
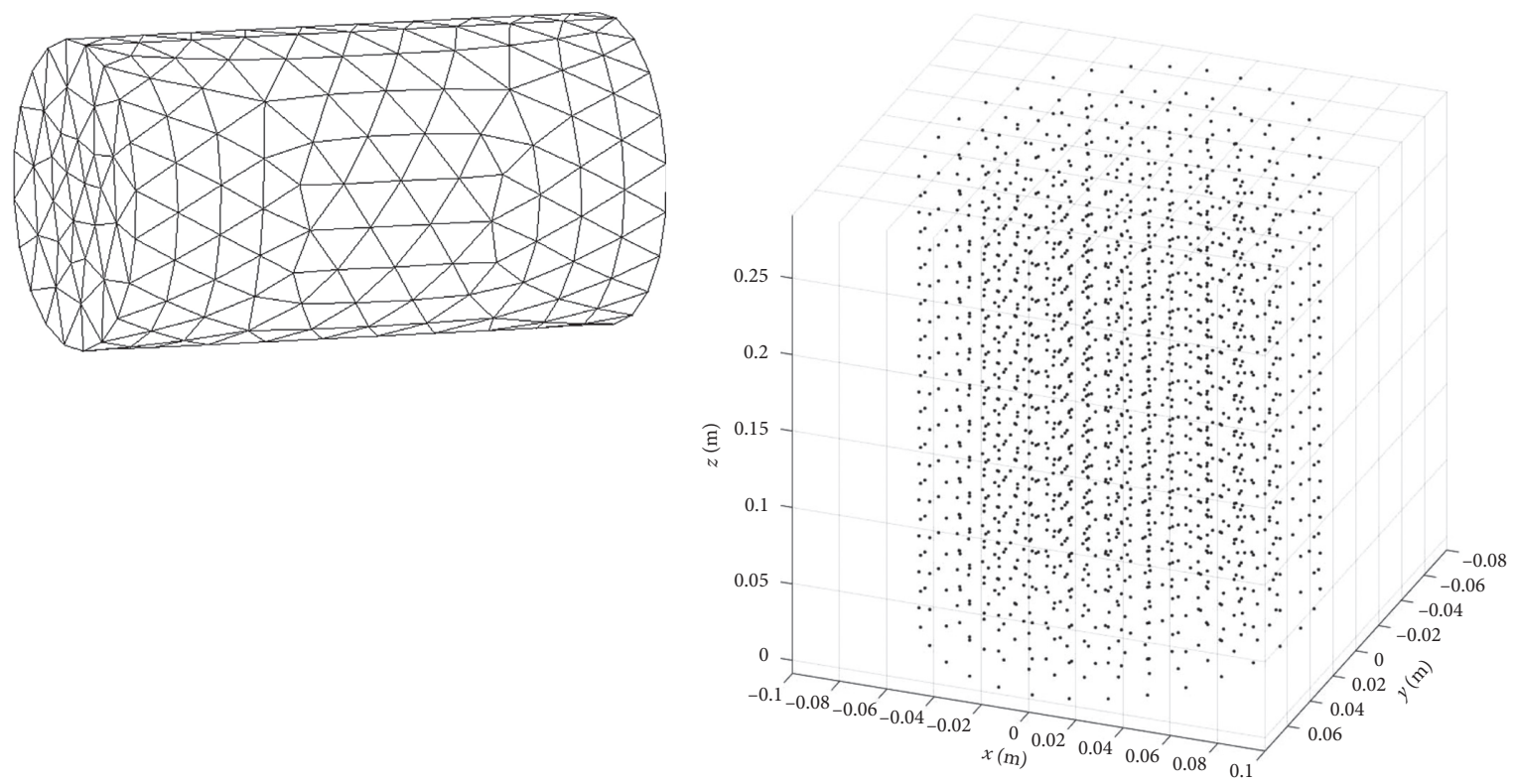

(a)

(b)

Figure 2: The distributions of the background mesh and the field nodes for calculating acoustic modes. (a) Mesh of background element. (b) Field nodes.

TABLE 2: Modal frequencies of expansion chamber from 3D weakform MFM and 3D FEM.

\begin{tabular}{lcccc}
\hline $\begin{array}{l}\text { Modal } \\
\text { order }\end{array}$ & & \multicolumn{2}{c}{ Modal frequencies } & $\begin{array}{c}\text { Relative } \\
\text { errors (\%) }\end{array}$ \\
\hline 1 & $(0,0,1)$ & 602.3 & 604.2 & 0.31 \\
2 & $(0,0,2)$ & 1204.2 & 1207.9 & 0.31 \\
3 & $(1,0,0)$ & 1307.7 & 1313.9 & 0.47 \\
4 & $(1,0,1)$ & 1441.2 & 1445.3 & 0.28 \\
5 & $(1,0,2)$ & 1778.9 & 1783.0 & 0.23 \\
6 & $(0,0,3)$ & 1805.1 & 1809.4 & 0.24 \\
7 & $(2,0,0)$ & 2157.2 & 2164.3 & 0.33 \\
8 & $(1,0,3)$ & 2229.9 & 2229.6 & 0.01 \\
9 & $(2,0,1)$ & 2239.6 & 2245.7 & 0.27 \\
10 & $(0,0,4)$ & 2402.5 & 2404.4 & 0.08 \\
11 & $(2,0,2)$ & 2470.5 & 2477.2 & 0.27 \\
12 & $(0,1,0)$ & 2735.3 & 2732.7 & 0.10 \\
13 & $(1,0,4)$ & 2736.0 & 2740.4 & 0.16 \\
14 & $(0,1,1)$ & 2776.5 & 2776.8 & 0.01 \\
15 & $(2,0,3)$ & 2812.8 & 2814.1 & 0.05 \\
\hline
\end{tabular}

In order to compare the computational speed of the $3 \mathrm{D}$ weak-form MFM method and 3D FEM used in this paper, the time required to calculate the first 15 order higher modes of the silencer displayed in Figure 1 is recorded. In the presented 3D weak-form MFM, the field nodes are created by using MATLAB codes, and the tetrahedral elements are used to form the background meshes by using the software package ANSYS, which are independent of the field nodes. In the 3D FEM, the codependent filed nodes and tetrahedral elements are used to build the $3 \mathrm{D}$ meshes by using the software package ANSYS, and the 3D acoustic modes are calculated by using the software package Virtual.Lab Acoustics. To achieve the same precision, 1775 nodes and 2505 background meshes are used in the presented MFM, and the computation time is $126 \mathrm{~s}$. By using the 3D FEM, the same 1775 nodes are used, since every node is contained in the element, and 8654 elements are required. It is worth noting that the time consumed by using the $3 \mathrm{D}$ FEM is $36 \mathrm{~s}$, less than when using the present weak-form MFM. It can be seen that, compared with the 3D FEM commercial software, the MFM computational codes used in this paper has no advantage in calculation speed. However, the computing advantage of the MFM will be displayed if the calculation model is more complicated. This is because, for complicated silencers, it needs more energy to divided high quality meshes using the FEM, and the filed nodes used in MFM are created by MALTAB codes, and the requirements on the background meshes are not so high, which will be less time-consuming. 


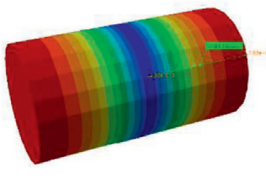

$(0,0,1)$

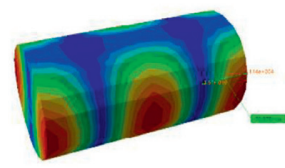

$(1,0,2)$

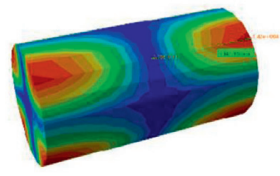

$(2,0,1)$

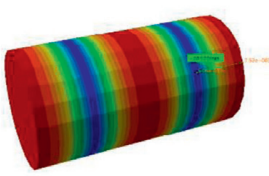

$(0,0,2)$

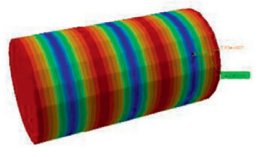

$(0,0,3)$

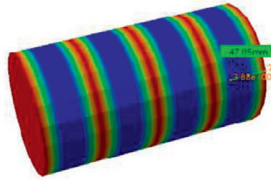

$(0,0,4)$

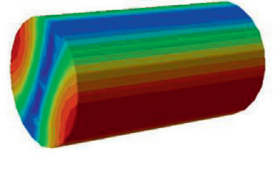

$(1,0,0)$

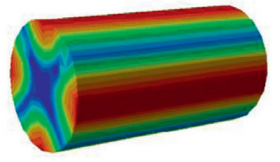

$(2,0,0)$

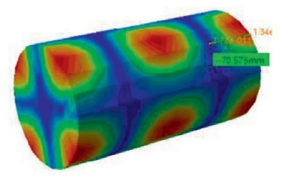

$(2,0,2)$

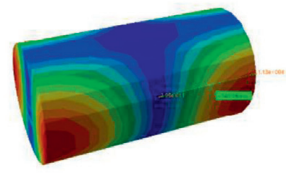

$(1,0,1)$

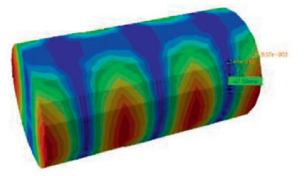

$(1,0,3)$

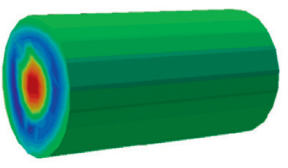

$(0,1,0)$

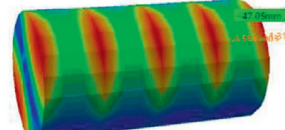

$(1,0,4)$

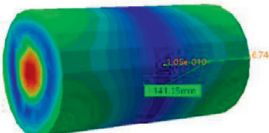

$(0,1,1)$

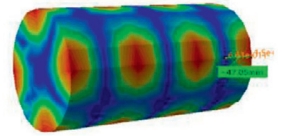

$(2,0,3)$

FIgURE 3: The TL of expansion chamber silencer from 3D MFM and analytical method.

By comparing the modal frequencies in Table 2 and the first 15 order modal shapes shown in Figure 3, it can be found that, due to the axisymmetric characteristics of the expansion chamber, all the circumferential modes are double root modes; that is, the modal frequencies are equal and the modal shapes are symmetrical about the central axis. Multiple root modes only listed one mode shape in Figure 4. The distance between all the axial nodal lines with the end of the expansion chamber are marked in Figure 4; it is worth noting that the nodal lines of the $(0,0,1),(1,0,1),(2,0,1)$, and $(0,1,1)$ modes locate at the centre of the expansion cavity axis, that is, $(1 / 2) L$. That is to say, the nodal lines of all higher-order modes including the first-order axial mode factor are located at $(1 / 2) L$. Similarly, all the nodal lines of all higher-order modes including the second-order axial mode factor are located at $(3 / 4) L$. For the $(0,0,3)$ and $(1,0,3)$ modes, there are three nodal lines, and one is located at $(1 / 2) L$, and the other two are located at $(5 / 6) L$. For the $(0$, $0,4)$ and $(1,0,4)$ modes, there are four nodal lines, and two are located at $(2 / 3) L$, and the other two are located at (5/6) L.

Figure 4 compares the transmission loss results of the expansion chamber silencer shown in Figure 1. The TL results are calculated by using the 3D weak-form MFM and analytical method. It can be seen from the comparisons that the TL results from the three methods agree well and the accuracy of the present 3D MFM for calculating TL of expansion chambers is invalidated.

Same as mode calculations, in the presented 3D weakform MFM, the field nodes are created by using MATLAB codes, and the tetrahedral elements are used to form the background meshes by using the software package ANSYS. In the 3D FEM, the codependent filed nodes and tetrahedral elements are used to build the $3 \mathrm{D}$ meshes by using the software package ANSYS, and the TL results are calculated by using the software package Virtual.Lab Acoustics. Due to the symmetry of the silencer, $(1 / 4)$ of the model is taken to compute TL.

In order to eliminate the influence of the number of nodes on the comparison results by using the global weakform MFM and the FEM, the number of nodes is approximately the same in the two methods. In FEM, the grid size is set as $0.01 \mathrm{~m}$ and the numbers of elements and nodes are 5325 and 2522, respectively. In MFM, the grid size is larger, that is, $0.015 \mathrm{~m}$, and the number of elements and nodes are 3908 and 2416.

In the case of approximate node number, the time used by MFM is $2643 \mathrm{~s}$, where the time of grid and node preparation is $500 \mathrm{~s}$, and the time of program calculation is $2143 \mathrm{~s}$. In the finite element method, $1621 \mathrm{~s}$ is needed, where $1321 \mathrm{~s}$ is needed for preprocessing work including the mesh and node preparation in ANSYS and parameter settings in 


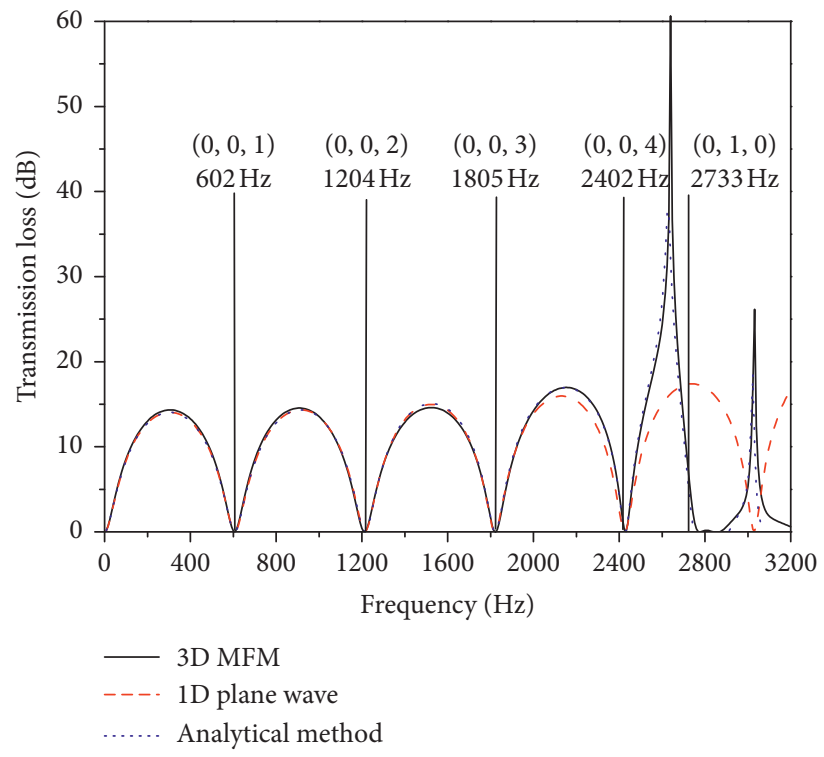

Figure 4: 3D modal shape of the expansion chamber.

TABLE 3: Modal frequencies for expansion chamber with extended inlet from 3D weak-form MFM and 3D FEM.

\begin{tabular}{lcccc}
\hline $\begin{array}{l}\text { Modal } \\
\text { order }\end{array}$ & & \multicolumn{2}{c}{ Modal frequencies } & $\begin{array}{c}\text { Relative } \\
\text { errors (\%) }\end{array}$ \\
\hline 1 & $(0,0,1)$ & 616.7 & 617.9 & 0.19 \\
2 & $(0,0,2)$ & 1208.9 & 1210.2 & 0.11 \\
3 & $(1,0,0)$ & 1236.3 & 1234.5 & 0.15 \\
4 & $(1,0,1)$ & 1405.1 & 1402.9 & 0.16 \\
5 & $(1,0,2)$ & 1768.3 & 1765.9 & 0.14 \\
6 & $(0,0,3)$ & 1821.5 & 1819.5 & 0.11 \\
7 & $(2,0,0)$ & 2162.4 & 2159.5 & 0.13 \\
8 & $(1,0,3)$ & 2233.1 & 2230 & 0.14 \\
9 & $(2,0,1)$ & 2248.6 & 2246.6 & 0.09 \\
10 & $(0,0,4)$ & 2444.8 & 2445.5 & 0.03 \\
11 & $(2,0,2)$ & 2500.1 & 2490.9 & 0.37 \\
12 & $(1,0,4)$ & 2756.1 & 2751.2 & 0.18 \\
13 & $(0,1,0)$ & 2767.2 & 2764.6 & 0.09 \\
14 & $(2,0,3)$ & 2843.4 & 2841.6 & 0.06 \\
15 & $(0,1,1)$ & 2930.2 & 2927.9 & 0.08 \\
\hline
\end{tabular}

Virtual.Lab Acoustic, while $300 \mathrm{~s}$ is needed for calculation. It can be seen that the finite element method has higher calculation efficiency for simple expansion chamber.

4.2. Expansion Chamber Silencer with Extended Inlet Duct. Then, the expansion chamber silencer with extended inlet is studied. The schematic diagram and size of the silencer are the same as expansion chamber shown in Figure 1. The only difference is that the extension of inlet duct is arranged as $L_{1}=0.08 \mathrm{~m}$. Then, the first 15 order modes and the TL results are computed.

In order to avoid the singular value caused by the wall of the inlet extension and the inaccuracy of the calculation result, the inlet duct is removed when calculating the acoustic modes and transmission loss. Since the sound is plane wave propagation in the inlet duct, this processing will not affect the calculation result. The mesh size and node distance are the same as those of the expansion chamber silencer. The MFM and the FEM use the same number of nodes to calculate the acoustic mode and TL, which is 1500 , while the numbers of meshes are 2405 and 5325, respectively. Table 3 and Figure 5 compare the first 15 order acoustic modal frequencies and transfer loss curves from the two methods. In the same computer, excluding the preprocessing time, the time of calculating three-dimensional acoustic modes and transmission loss by MFM is $150 \mathrm{~s}$ and $2240 \mathrm{~s}$, respectively, while that of FEM is $40 \mathrm{~s}$ and $400 \mathrm{~s}$. It can be seen that the two methods can achieve the same accuracy when using the same nodes, but the meshless method requires relatively long calculation time.

4.3. Comparison of Computational Time. As the regularly shaped section has exact analytical modal frequencies, the simple expansion chamber shown in Figure 1 is investigated to compare the computational efficiency of the 3D MFM. For convenience of analysis, the relative error of the $3 \mathrm{D}$ modal frequencies is defined as follows:

$$
\delta=\frac{\left|f-f^{\text {Exact }}\right|}{f^{\text {Exact }}} \times 100(\%),
$$

where $f$ and $f^{\text {Exact }}$ are the modal frequencies obtained from the numerical and analytical methods, respectively.

It can be seen that only the transversal modal frequencies have exact analytical solutions, and the axial modal frequencies is equal to the pass through frequencies of the expansion chamber, which can be obtained by the following equation:

$$
f_{n}=n \frac{c}{2 L}, \quad(n=1,2,3, \ldots)
$$




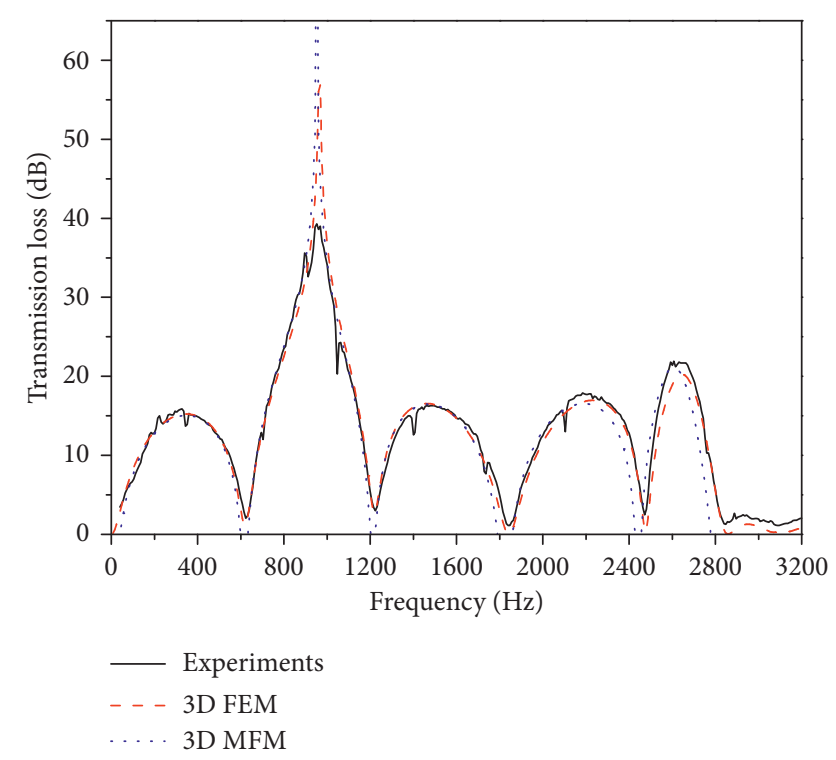

FIGURE 5: The TL results of expansion chamber silencer with inlet extension from the 3D MFM, 3D FEM, and experimental measurement.

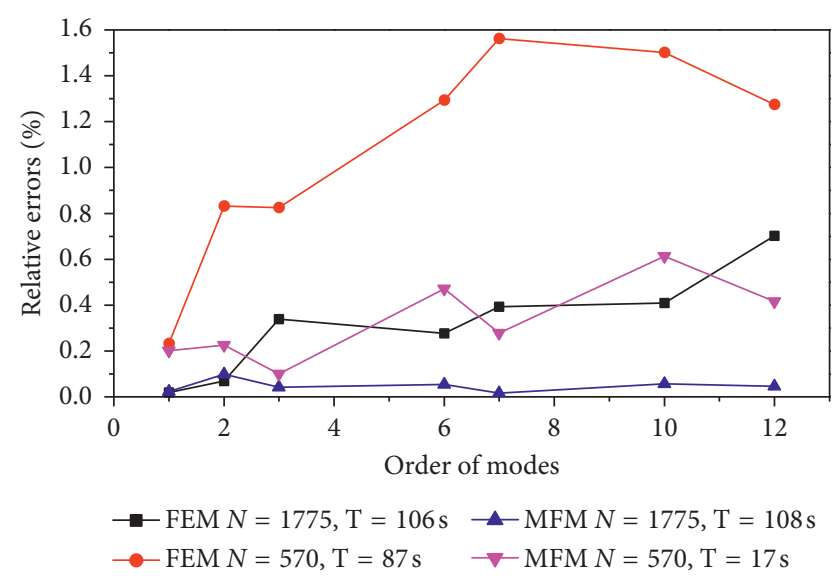

FIGURE 6: Comparison of the computational efficiency of MFM and FEM.

where $L$ is the length of the chamber. Therefore, the $1^{\text {st }}, 2^{\text {nd }}$, $3^{\text {rd }}, 6^{\text {th }}, 7^{\text {th }}, 10^{\text {th }}$, and $12^{\text {th }}$ modes have analytical modal frequencies below $3000 \mathrm{~Hz}$ for the expansion chamber silencer.

Figure 6 gives the relative error of the transversal and axial modal frequencies from the MFM and the FEM with different numbers of field nodes. To constrain the relative error within $0.6 \%$, the weak-form MFM requires 570 field nodes, and the computational time is $17 \mathrm{~s}$; the FEM requires 1775 field nodes, and the computational time is $106 \mathrm{~s}$. Most of the time in the finite element method is used for preprocessing, such as reading model and setting material parameters, which is $70 \mathrm{~s}$, and the time used for calculating modes is just $36 \mathrm{~s}$. Since less background grids are needed, the MFM is more time saving. With 570 field nodes, the FEM exhibit worse precision, and the maximum relative error is approximately $1.6 \%$, and the computational time is $87 \mathrm{~s}$, where $70 \mathrm{~s}$ is used for preprocessing and $17 \mathrm{~s}$ is used for calculating modes.

All computations are conducted on the same computer. It can be concluded that, in order to get the same calculation precision, 3D MFM needs less filed nodes and background elements and more time saving than 3D FEM, especially for complex configurations.

\section{Results and Discussions}

Acoustic modes are the special characteristics of the silencer, and the acoustic performance can be analyzed according to the modal features. In order to facilitate the analysis, the transmission loss of the expansion chamber calculated using the one-dimensional plane wave theory is also given in Figure 3, and the end correction of the inlet and outlet ducts is considered in this calculation [1]. Comparing the TL results from the $1 \mathrm{D}$ and $3 \mathrm{D}$ methods, it can be seen that the two curves deviate significantly at the frequency of the $(0,1,0)$ order mode (namely, $2733 \mathrm{~Hz}$ ), and the 3D weak-from mesh-free calculation curve no longer has arched characteristics, indicating that a transversal higher-order mode appears in the expansion cavity. Because the effects of transversal modes are not considered in the one-dimensional plane wave theory, the calculation results are no longer accurate. Since the end correction of the inlet and outlet and the effect of the axial higher-order modes both are considered in the plane wave theory, the two curves match well in the frequency range below the $(0,0,4)$ order modal frequency (namely, $2408 \mathrm{~Hz}$ ). It can be concluded that the influence of the dissipative three-dimensional wave caused by the abrupt cross section at the inlet and outlet sections of the silencer is also considered in the one-dimensional plane wave theory.

Since the circumferential mode is symmetrical about the central axis and the inlet and outlet ducts are located on the central axis, none of the circumferential modes are excited. The first excited mode is the first-order radial mode. The acoustic attenuation frequency range can be widened by locating the outlet duct on the radial modal line, which has been analyzed in the existing literature [1]. It can also be found from the transmission loss curve that the pass frequency of the silencer corresponds to the axial high-order modal frequency of the expansion cavity, so in order to eliminate the pass frequency, in addition to inlet/outlet extension [1], the outlet tube can also be arranged on the axial nodal line, in the form of a side branch outlet. It can be seen from the modal shape diagrams shown in Figure 4 that the nodal lines of the $(0,0,1)$ and $(0,0,2)$ order modes are located at (1/2), (1/4), and (3/4) of the expansion cavity length, respectively. It is conceived that the effects of the corresponding axial modes can be eliminated by setting the outlet duct at these positions, respectively; namely, the pass frequencies can be eliminated.

5.1. Effects of Side Branch Outlet on TL of Expansion Chamber Silencer. In this paper, the TL results of two silencers (namely, silencer $\# a$ and silencer $\# b$ ) are calculated to validate the effects of the acoustic modes on the acoustic 


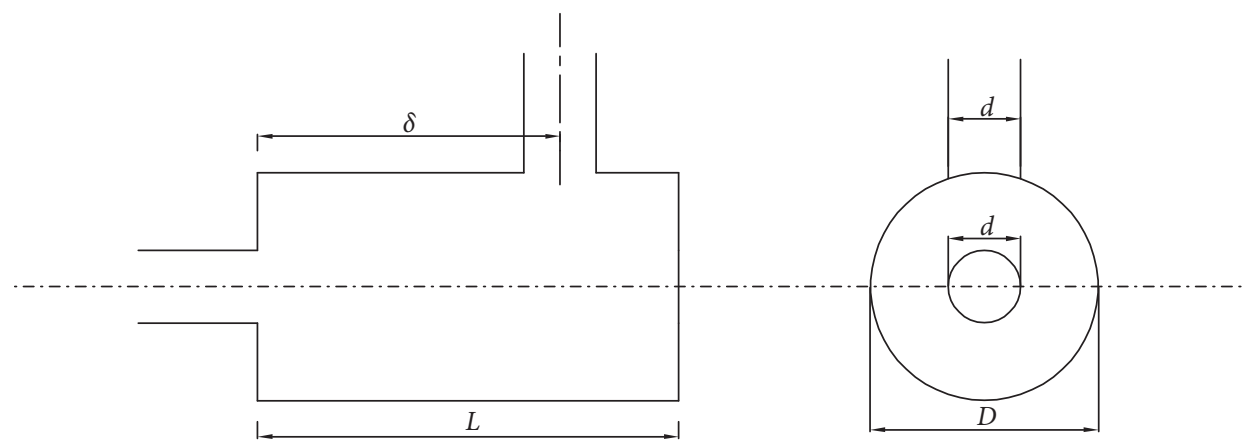

FIGURE 7: The schematic diagram of the expansion chamber with side branch outlet.

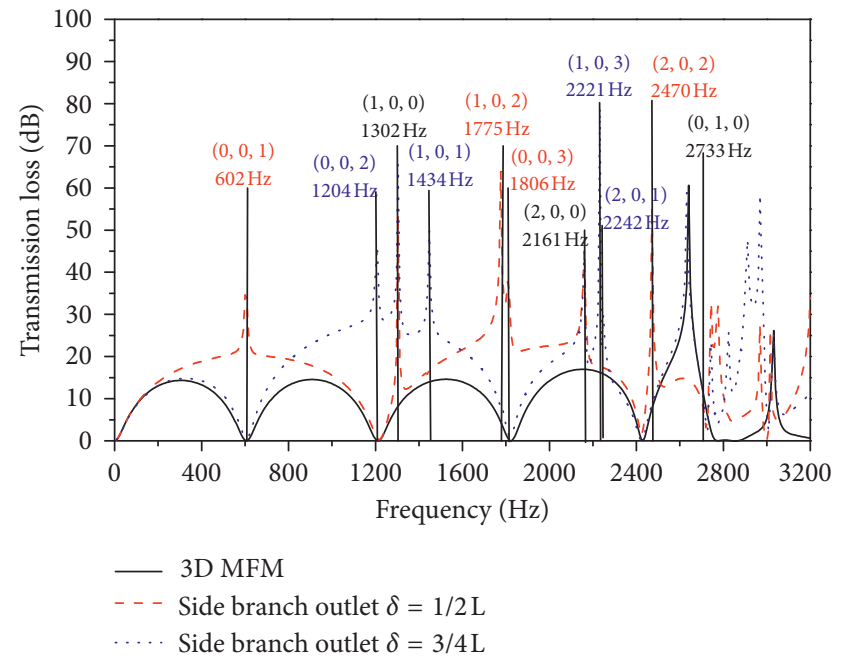

FIgURE 8: Location of side branch outlet on TL of expansion chamber.

attenuation performance. The outlet tube of the two silencers is arranged at (1/2) and (3/4) of the length of the expansion cavity, respectively. The schematic diagram of the expansion chamber with side branch outlet is shown in Figure 7. The offsets of the outlet duct are taken $\delta=(L / 2)=0.14115 \mathrm{~m}$ for silencer $\# a$ and $\delta=(3 / 4) L=0.211725 \mathrm{~m}$ for silencer $\# b$, separately, and other dimensions are the same as those in Figure 1. The transmission loss curves calculated by the present 3D weak-form MFM are given in Figure 8. In order to distinguish the modes more clearly, in Figure 8, the modes eliminated by the silencer $\# a$ are noted by red words, and the modes eliminated by the silencer $\# b$ are noted by blue words, and the modes eliminated by both the silencer $\# a$ and silencer $\# b$ are noted by black words. For the silencer $\# a$, the side branch outlet duct is located on the $(0,0,1)$ modal line, so a peak appears at the modal frequency $(602 \mathrm{~Hz})$ in the transmission loss curve. Compared with the silencer with coaxial inlet and outlet ducts, the first pass frequency is eliminated. It can be observed that the influence of the thirdorder axial mode $((0,0,3)$ mode $)$ is also eliminated. This is because one of the nodal lines of the $(0,0,3)$ mode is also located at $(L / 2)$. Similarly, for the silencer \#b, the outlet pipe is located on the $(0,0,2)$ nodal line, and the effect of $(0,0,2)$ order mode is removed, exhibiting better acoustic attenuation performance at $(0,0,2)$ modal frequency. It can be seen that peaks in the TL curves for the two silencers appear at the two circumferential modal frequencies of $(1,0,0)$ and $(2,0,0)$, that is, $1302 \mathrm{~Hz}$ and $2161 \mathrm{~Hz}$, and the silencer $\# a$ also exhibits better acoustic performance at $(1,0$, $2)$ and $(2,0,2)$ modal frequencies, and silencer $\# b$ also exhibits better acoustic performance at $(1,0,1)$ and $(2,0,1)$ modal frequencies, where transmission loss peaks appear. This is because whether the outlet pipe is located at $(1 / 2) L$ or (3/4) $L$, the outlet duct is located on the nodal lines of the modes including the two circumferential modes of $(1,0,0)$ and $(2,0,0)$, and so the effects of the circumferential modes can be eliminated. However, when the axial modes $(0,0,1)$ exist at the same time, that is, all the nodal lines of modes (1, $0,1)$ and $(2,0,1)$ intersecting at $(1 / 2) L$, where the sound pressure is zero on a large area, rather than the simple intersection of nodal lines, the outlet pipe at $(1 / 2) L$ cannot eliminate the influence of this mode. On the contrary, the outlet pipe at (1/4) $L$ or (3/4) $L$ can eliminate the influence of this mode. Therefore, the transmission loss peaks appear at the $(1,0,1)$ and $(2,0,1)$ modal frequencies (namely, $1434 \mathrm{~Hz}$ and $2234 \mathrm{~Hz}$ ) for silencer \#b. Similarly, for silencer $\# a$, the outlet is located on the $(1 / 2) L$ and can eliminate the effects of the $(1,0,2)$ and $(2,0,2)$ order modes. So, it can be observed that there are attenuation peaks at $1775 \mathrm{~Hz}$ and $2740 \mathrm{~Hz}$ in the transmission loss curve. It can be seen from the modal shapes that the amplitude of sound pressure is not zero on the nodal line of $(0,0,4)$ mode, which is the same order of magnitude as the maximum value of sound pressure. Therefore, although the outlet pipe is placed on the modal nodal line of this order mode, the influence of this order mode has not been eliminated, so there is no peak near $2400 \mathrm{~Hz}$ on the transmission loss curves; that is, the fourth passing frequency has not been eliminated. It can be concluded that, compared with coaxial silencer, the influence of a certain axial mode can be eliminated and the acoustic attenuation performance can be greatly optimized by arranging the side branch outlet duct at the nodal line of this axial mode.

5.2. Effects of Side Branch Outlet on TL of Expansion Chamber Silencer with Extended Inlet. Similarly, the effects of acoustic modes on the acoustic attenuation performance of expansion chamber with extended inlet shown in Figure 9 are 


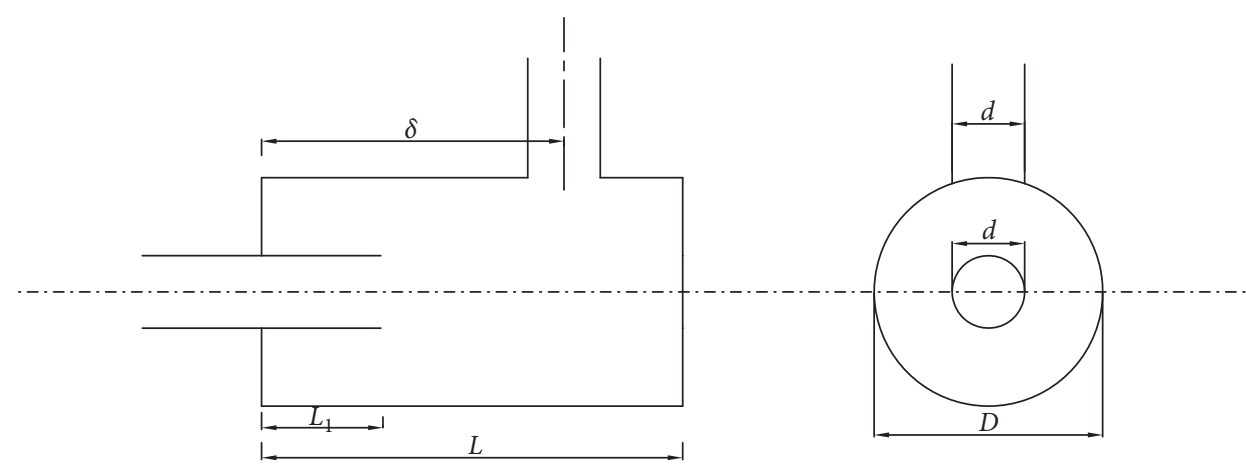

FIgURE 9: The schematic diagram of the expansion chamber with extended inlet and side branch outlet.

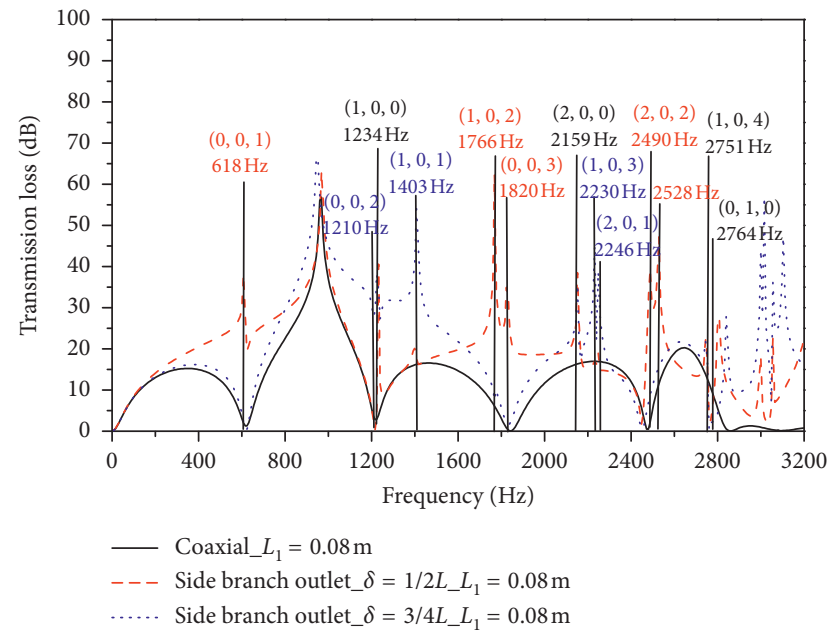

FIGURE 10: Location of side branch outlet on TL of expansion chamber with extended inlet outlet.

investigated in Figure 10. The modal shapes of the 3D acoustic modes of expansion chamber with extended inlet are the same as that of expansion chamber shown in Figure 4, and only the modal frequencies are different. Therefore, the outlet are located on the same location (namely, $\delta=(L / 2)=0.14115 \mathrm{~m}$ and $\delta=(3 / 4) L=$ $0.211725 \mathrm{~m}$ ). It can be seen from Figure 10 that the effects of the location of outlets on the acoustic attenuation performance is the same as that of the expansion chamber. Locating the outlet on the $(1 / 2) L$ can eliminate the effects of the $(0,0,1),(1,0,2),(0,0,3)$, and $(2,0,2)$ order modes, and the attenuation peaks appear at these modal frequencies in the TL curves. Locating the outlet on the (3/4) $L$ can eliminate the effects of the $(0,0,2),(1,0,1),(1,0,3)$, and $(2$, $0,1)$ order modes, and the attenuation peaks appear at these modal frequencies in the TL curves. The reasons are the same as the analysis of the expansion chamber without extensions. The effects of the $(1,0,0)$ and $(2,0,0)$ modes are eliminated by the two configurations, and this is because the inlet is located on the two modal lines.

\section{Conclusions}

A global weak-form MFM based on the RPI-Galerkin method has been proposed to evaluate the $3 \mathrm{D}$ acoustic modes and transmission loss of expansion chamber silencers. In extracting the acoustic eigenvalues (modal frequencies) and eigenvectors (pressure modes) as well as the transmission loss by using the weak-form MFM, the RPIM is used to construct the shape function, the Galerkin method is applied to develop the discretized system equation, and the Gauss integration scheme is carried out to calculate system matrices. The acoustic modal frequencies and TL of the expansion chamber silencers from the proposed 3D weak-form MFM are compared with the results from 3D finite element method and analytical or experimental data. Based on the test examples, it can be concluded that the MFM for solving the $3 \mathrm{D}$ acoustic modefs and TL are independent of the element topology and require fewer background elements. Additionally, the effects of axial modes on the acoustic characteristics of expansion chamber silencers are investigated, and the pass through frequencies can be eliminated to enhance the acoustic attenuation performance by locating the side branch outlet on the nodal lines of axial modes.

\section{Data Availability}

The numerical data used to support the findings of this study are included within the article.

\section{Conflicts of Interest}

The authors declare that they have no conflicts of interest.

\section{Acknowledgments}

The authors acknowledge the support of the Fundamental Research Funds for the Central Universities (grant no. 2018KFYYXJJ3).

\section{References}

[1] A. Selamet and Z. L. Ji, "Acoustic attenuation performance of circular expansion chamber with extended inlet/outlet," Journal of Sound and Vibration, vol. 223, no. 2, pp. 197-212, 1998.

[2] F. D. Denia, J. Albelda, F. J. Fuenmayor, and A. J. Torregrosa, "Acoustic behaviour of elliptic chamber silencers," Journal of Sound and Vibration, vol. 241, pp. 401-421, 2000. 
[3] A. Selamet, M. B. Xu, I.-J. Lee, and N. T. Huff, "Analytical approach for sound attenuation in perforated dissipative silencers with inlet/outlet extensions," The Journal of the Acoustical Society of America, vol. 117, no. 4, pp. 2078-2089, 2005.

[4] R. Kirby and F. D. Denia, "Analytic mode matching for a circular dissipative silencer containing mean flow and a perforated pipe," The Journal of the Acoustical Society of America, vol. 122, no. 6, pp. 3471-3482, 2007.

[5] R. Kirby, "Transmission loss predictions for dissipative silencers of arbitrary cross section in the presence of mean flow," The Journal of the Acoustical Society of America, vol. 114, no. 1, pp. 200-209, 2003.

[6] F. D. Denia, E. M. Sánchez-Orgaz, L. Baeza, and R. Kirby, "Point collocation scheme in silencers with temperature gradient and mean flow," Journal of Computational and Applied Mathematics, vol. 291, pp. 127-141, 2016.

[7] J. Albelda, F. D. Denia, M. I. Torres, and F. J. Fuenmayor, “A transversal substructuring mode matching method applied to the acoustic analysis of dissipative mufflers," Journal of Sound and Vibration, vol. 303, no. 3-5, pp. 614-631, 2007.

[8] R. Kirby, "A comparison between analytic and numerical methods for modelling automotive dissipative silencers with mean flow," Journal of Sound and Vibration, vol. 325, no. 3, pp. 565-582, 2009.

[9] Z. Fang and Z. L. Ji, "Finite element analysis of transversal modes and acoustic attenuation characteristics of perforated tube silencers," Noise Control Engineering Journal, vol. 60, no. 3, pp. 340-349, 2012.

[10] Z. Fang and Z. L. Ji, "Acoustic attenuation analysis of expansion chambers with extended inlet/outlet," Noise Control Engineering Journal, vol. 61, no. 2, pp. 240-249, 2013.

[11] Z. Fang and Z. L. Ji, "Numerical mode matching approach for acoustic attenuation predictions of double-chamber perforated tube dissipative silencers with mean flow," Journal of Computational Acoustics, vol. 22, no. 2, 2014.

[12] Z. Fang, Z. L. Ji, and C. Y. Liu, "Acoustic attenuation analysis of silencers with multi-chamber by using coupling method based on subdomain division technique on subdomain division technique," Applied Acoustics, vol. 116, pp. 152-163, 2017.

[13] A. Karageorghis, "The method of fundamental solutions for the calculation of the eigenvalues of the Helmholtz equation," Applied Mathematics Letters, vol. 14, no. 7, pp. 837-842, 2001.

[14] C. Wentreodt and O. Von Estorff, "Dispersion analysis of the meshfree radial point interpolation method for the Helmholtz equation," International Journal for Numerical Methods in Engineering, vol. 77, no. 12, pp. 1670-1689, 2009.

[15] K. Li, Q. B. Huang, J. L. Wang, and L. G. Lin, “An improved localized radial basis function meshless method for computational aeroacoustics," Engineering Analysis with Boundary Elements, vol. 35, no. 1, pp. 47-55, 2011.

[16] Z. He, P. Li, G. Y. Zhao, and H. Chen, "A meshless Galerkin least-square method for the Helmholtz equation," Engineering Analysis with Boundary Elements, vol. 35, no. 6, pp. 868-878, 2011.

[17] L. Y. Yao, D. J. Yu, and J. W. Zhou, "Numerical treatment of $2 \mathrm{D}$ acoustic problems with the cell-based smoothed radial point interpolation method," Applied Acoustics, vol. 73, no. 67, pp. 557-574, 2012.

[18] L. Wang, F. Chu, and Z. Zhong, "Study of radial basis collocation method for wave propagation," Engineering Analysis with Boundary Elements, vol. 37, no. 2, pp. 453-463, 2013.
[19] Z. Fang and C. Y. Liu, "Combined mesh free method and mode matching approach for transmission loss predictions of expansion chamber silencers," Engineering Analysis with Boundary Elements, vol. 84, pp. 168-177, 2017.

[20] Z. Fang and C. Y. Liu, "Semi-weak-form mesh-free method for acoustic attenuation analysis of silencers with arbitrary but axially uniform transversal sections," Journal of Sound and Vibration, vol. 442, pp. 752-769, 2019.

[21] M. L. Munjal, Acoustics of Ducts and Silencers, John Wiley \& Sons, Chichester, UK, 2nd edition, 2014.

[22] J. G. Wang and G. R. Liu, "On the optimal shape parameters of radial basis functions used for 2-D meshless methods," Computer Methods in Applied Mechanics and Engineering, vol. 191, no. 23-24, pp. 2611-2630, 2002.

[23] R. G. Liu, Mesh Free Methods: Moving Beyond the Finite Element Method, CRC Press, Boca Raton, FA, USA, 2003. 